\title{
UWB with Gain Enhancement Archimedean Spiral Microstrip serial Array Antennas for On-board Satellite Communications
}

\author{
Mahmoud Rajab \\ Faculty of engineering, Ain- \\ Shams University, Cairo, Egypt
}

\author{
Fatma El-Hefnawi \\ NARSS, ERI, Cairo, Egypt
}

\author{
Salwa $\mathrm{H}$. Elramly \\ Faculty of engineering, Ain- \\ Shams University, Cairo, Egypt
}

\begin{abstract}
Microstrip Archimedean spiral antennas are suitable for wideband satellite communications when CubeSat is used because of its light weight and small size. The wide band frequency response of the spiral antennas can be achieved by properly choosing their outer and inner radius .In this paper a spiral microstrip antennas are designed on FR-4, giving an ultra-wide band from 6 -to- $22 \mathrm{GHz}$ which covers all working satellite bands. High gain directive beam with suitable radiations in multiple frequencies for on-board satellite communications is obtained.
\end{abstract}

\section{Keywords}

UWB, Serial Array, DASMA, RT 5880, Gain enhancement

\section{INTRODUCTION}

Spiral antenna is widely used in satellite communications due to its wide band frequency response, the circular polarization, and the directive beam [1]-[5]. The continuous change in the diameter along the spiral radiating elements provides the smooth variation of the operating frequency response [3]. For this type of spiral configuration, the smaller and bigger diameter determines the high frequency and the low frequency of the operating band respectively. Traditionally to make the spiral radiating only in a one half-space, it is imperative to fill cavities by absorbing materials. In spite of cavities absorb the back radiation from the spiral and keeping the bandwidth features of the antenna, it suffers from certain limitations, including the $3 \mathrm{~dB}$ gain reduction, reducing the available radiation power by $50 \%$ and the extended volume occupied of the metallic cavity filled by a properly absorbing material. For these reasons, the hot area of research on spiral antennas is the possibility of having spirals on a substrate directly stacked over a metallic ground plane, without absorbers and cavities. This design may lead to overcome the previous disadvantages. In fact, the ground plane produces unwanted reflections, which may effect on the radiation pattern shape and limiting the bandwidth of operation. Choosing the substrate of suitable dielectric constant to the space environment FR-4 is chosen [6]-[10]. Six antenna designs are presented in this paper. The first antenna design is a dipole Archimedean spiral microstrip antenna DASMA on FR-4 substrate and ground plane forms UWB circular polarization which is suitable for on-board satellite communications with the earth station. The second antenna design is a dipole Archimedean spiral microstrip antenna DASMA on FR-4 substrate stacked on RT 5880 substrate with ground plane which is giving more gain and circular polarization with directive beam which increases the visibility duration with the earth station [11]. The third antenna design is a $2 \times 2$ dipole
Archimedean spiral microstrip array antenna DASMA on FR4 substrate and ground plane. The fourth antenna design is a 2x2 dipole Archimedean spiral microstrip array antenna on FR-4 stacked on RT 5880 substrate with ground plane. The fifth antenna design is a $3 \times 2$ Archimedean spiral microstrip serial array antenna on FR-4 substrate and ground plane. The sixth antenna design is a $3 \times 2$ Archimedean spiral microstrip serial array antenna on FR-4 stacked on RT 5880 substrate with ground plane. All antenna designs presented in this paper demonstrated without using cavities or absorbing materials since these may reduce the bandwidth and distort the radiation pattern, [12]-[14]. The simulation processes are performed using the CST microwave studio simulator. The measurements of the S-parameters are recorded using ROHDE \& SCHWAEZ ZVA 67 vector network analyzer. The manufacturing is performed at ERI.

\section{DIPOLE ARCHIMEDEAN SPIRAL MICROSTRIP ANTENNA DASMA DESIGN WITH GAIN ENHANCEMENT}

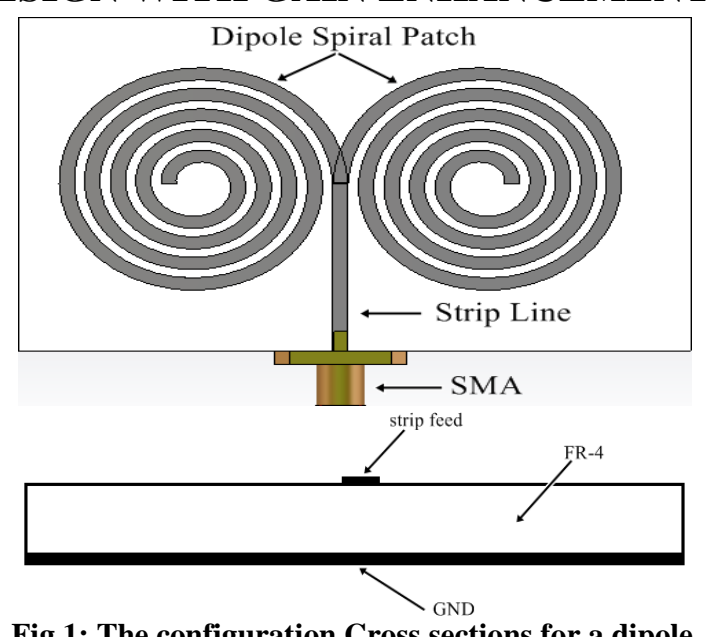

Fig 1: The configuration Cross sections for a dipole Archimedean spiral microstrip antenna DASMA without RT 5880 layer

The dipole Archimedean spiral patches designed with four spiral turns as a dipole which is represented in Fig. (1, and printed on a FR-4 substrate with $\left(\varepsilon_{\mathrm{r}}=4.7\right)$ with a height $\mathrm{h}=0.75 \mathrm{~mm}$ stacked on the infinite metallic ground plane .The dimension of spiral patch center diameter $D_{c}$ is $2 \mathrm{~mm}$, the thickness of spiral arm width $\mathrm{T}_{\mathrm{w}}$ is $1.47 \mathrm{~mm}$ the separation between the arms $T_{s}$ is $1 \mathrm{~mm}$ with strip line feed on the substrate surface with same dimension of spiral arm width After doing optimization and parameterization on the 
geometrical dimensions and taking into account the available material and fabrication techniques, the antenna with this design operate in the band between 7.5 -to- $24 \mathrm{GHz}$ as shown in Fig. (2) [15]. Circular polarization achieved with this antenna as shown in figure (3) having an axial ratio below $3 \mathrm{~dB}$. The gain is too low, as shown in figure (4) which need to increase to be suitable for on-board communications so stacked substrates gain enhancement technique can be used to raise the gain and radiation direction [16]-[20].

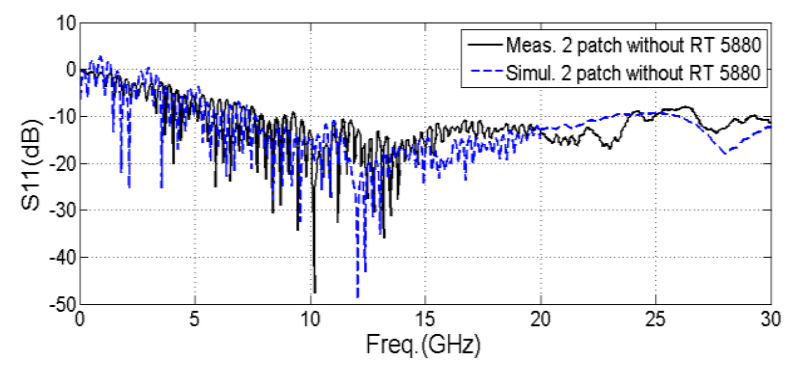

Fig 2: The return loss for a dipole Archimedean spiral microstrip antenna DASMA without RT 5880 layer.

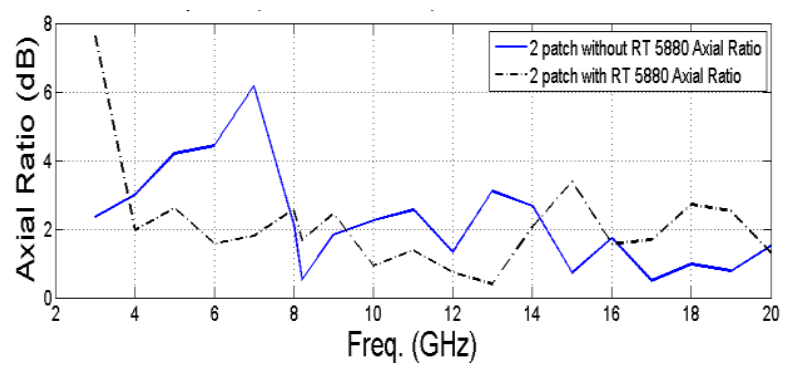

Fig 3: The axial ratio in broadband form for a dipole Archimedean spiral microstrip antenna DASMA with and without RT 5880 layer

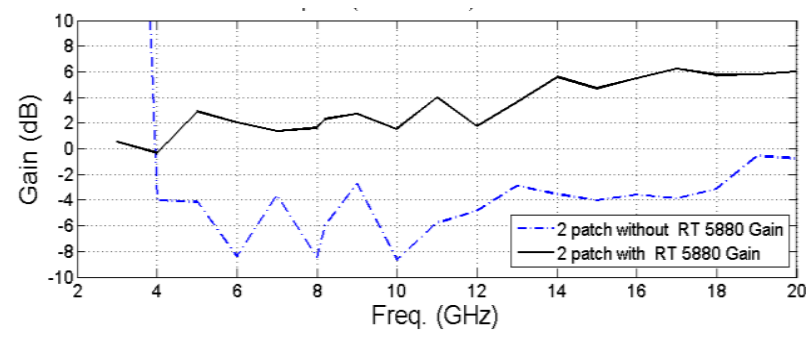

Fig 4: Gain in broadband form for a dipole Archimedean spiral microstrip antenna DASMA with and without RT 5880 layer

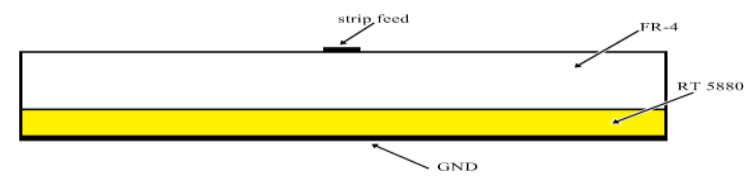

Fig 5: The configuration of the cross section for a dipole Archimedean spiral microstrip antenna DASMA with RT 5880 layer

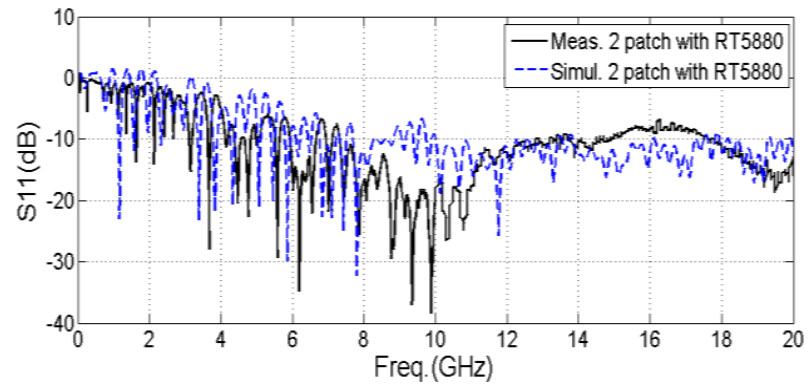

Fig 6: The return loss for a dipole Archimedean spiral microstrip antenna DASMA with RT 5880 layer

Performing the enhancement technique on the dipole configuration shown in Fig. (5). The frequency band of operation is decreased between $7.5-15 \mathrm{GHz}$ with approximately $7 \mathrm{GHz}$ working band as shown in Fig. (6). The polarization of the new design is circular as shown in Fig. (3) with an axial ratio below $3 \mathrm{~dB}$. This design has narrower band than the previous design, because of the mutual induction phenomena. Radiation patterns at multiple frequencies presented in polar and 3D forms are shown in figure (7), which forms an isoflux radiation shapes. On the other hand the antennas gain shown in Fig. (4) Increases by $+10 \mathrm{~dB}$ relative to the normal DASMA where no stacked RT 5880 layer is employed. The fabrication shown in Fig. (8).

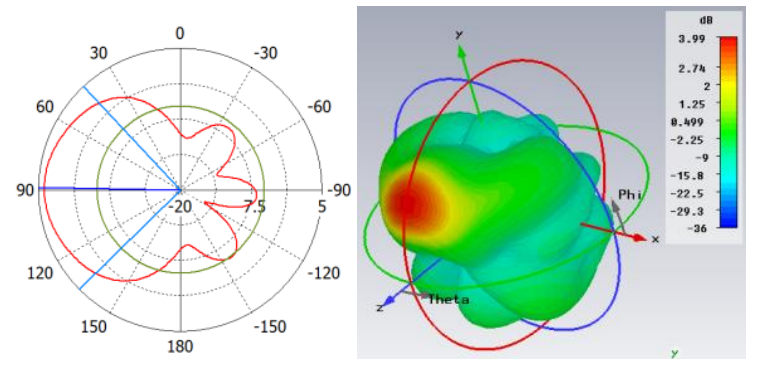

(a)

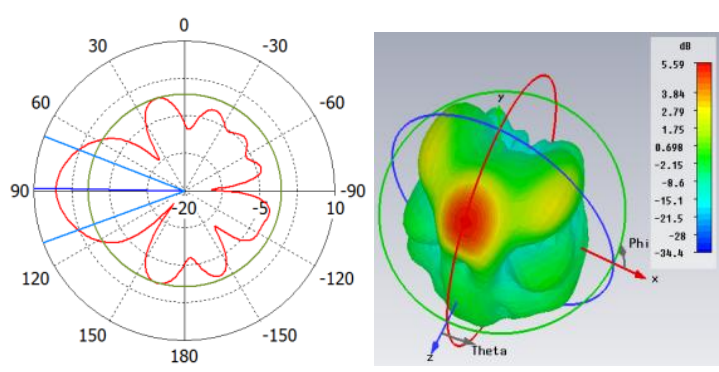

(b)

Fig 7: The gain in polar and 3D form in multiple frequencies for a dipole Archimedean spiral microstrip antenna DASMA with RT 5880 layer. (a) At frequency $11 \mathrm{GHz}$ with gain $4 \mathrm{~dB}$. (b) At frequency $14 \mathrm{GHz}$ with gain $6 \mathrm{~dB}$ 


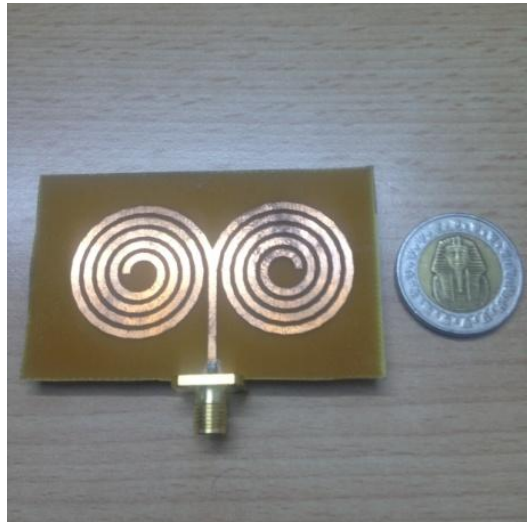

Fig 8: The fabricated DASMA photo

\section{SERIAL ARRAY DIPOLE ARCHIMEDEAN SPIRAL MICROSTRIP ANTENNA $2 \times 2$ DASMA DESIGN AND GAIN ENHANCEMENT}

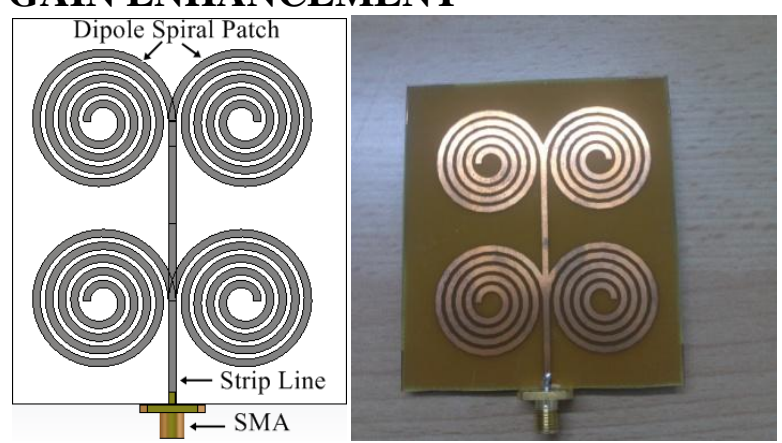

Fig 9: The cross section for $2 \times 2$ DASMA without RT 5880 layer and its fabricated photo

The modified design of the dipole Archimedean spiral microstrip antenna DASMA is represented by adding another dipole in series forming an array. The four patches are printed on FR-4 stacked directly to the ground plane as shown in Fig. (9). This new antenna array design operates in the band between 7.5-to- $25 \mathrm{GHz}$ as shown in Fig. (10). Circular polarization is achieved having an axial ratio below $3 \mathrm{~dB}$ as shown in Fig. (11), and a gain over $0 \mathrm{~dB}$; depicted in figure (12).More enhancement is employed by adding stacked substrate that improves the gain [16]-[20].

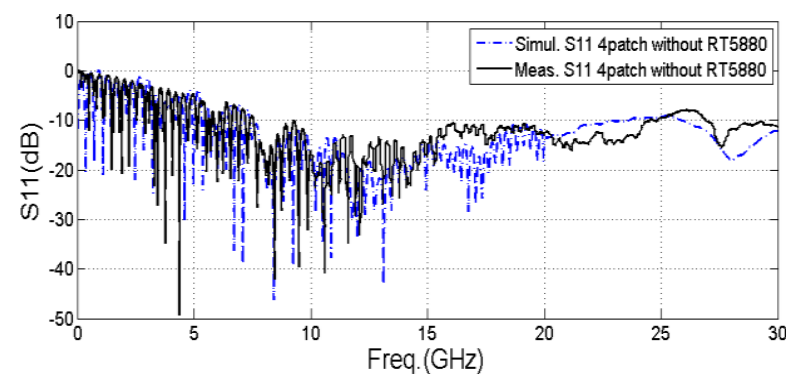

Fig 10: The return loss for 2x2 DASMA without RT 5880 layer

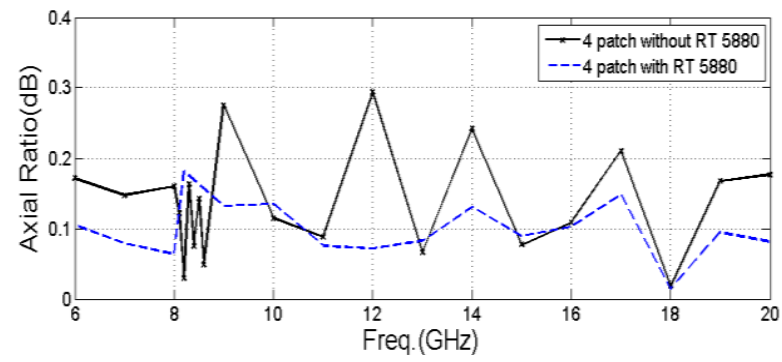

Fig 11: The axial ratio in broadband form for $2 \times 2$ DASMA with VS without RT 5880 layer

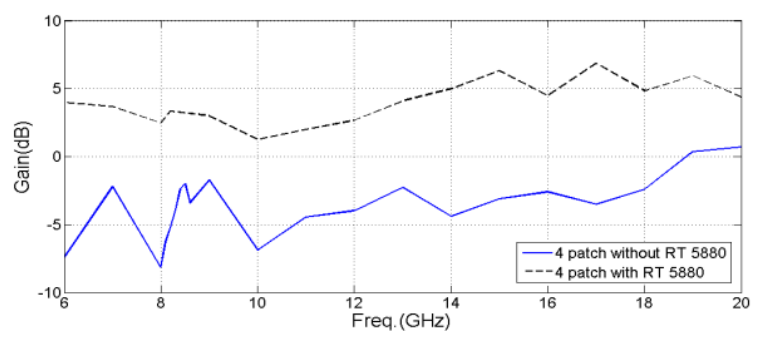

Fig 12: The gain in broadband form for $2 \times 2$ dipole Archimedean spiral microstrip antenna DASMA with and without RT 5880 layer

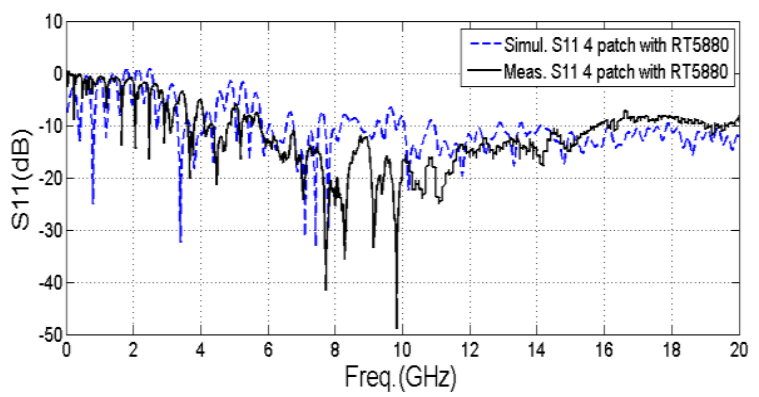

Fig 13: The return loss for $2 \times 2$ dipole Archimedean spiral microstrip antenna DASMA with RT 5880 layer

The new array design with the stacked substrates technique is shown in Fig. (5).This new geometry increases the thickness of FR-4 substrate to $1.5 \mathrm{~mm}$; adding RT 5880 layer $\left(\varepsilon_{\mathrm{r}}=2.2\right)$ giving a new height of $(0.508 \mathrm{~mm})$. Thus the total substrate height becomes $2.008 \mathrm{~mm}$. The new antenna design will operate between 5.8 -to- $16 \mathrm{GHz}$ forming a width of $10.2 \mathrm{GHz}$ with circular polarization as shown in Fig. (13) and Fig. (11) respectively

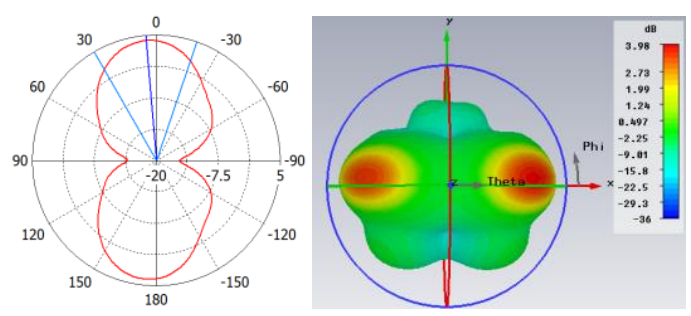

(a) 


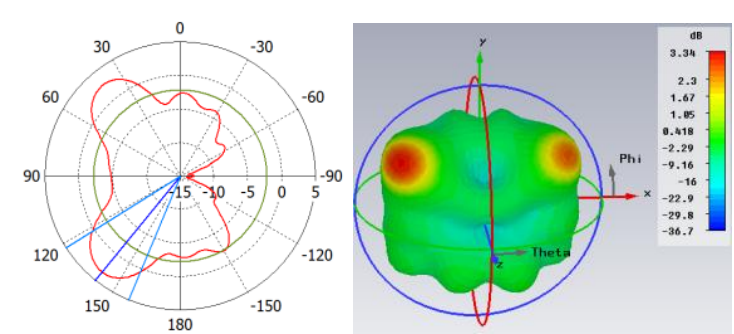

(b)

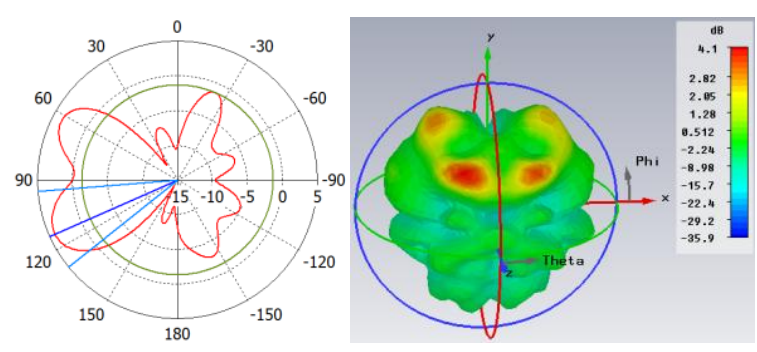

(c)

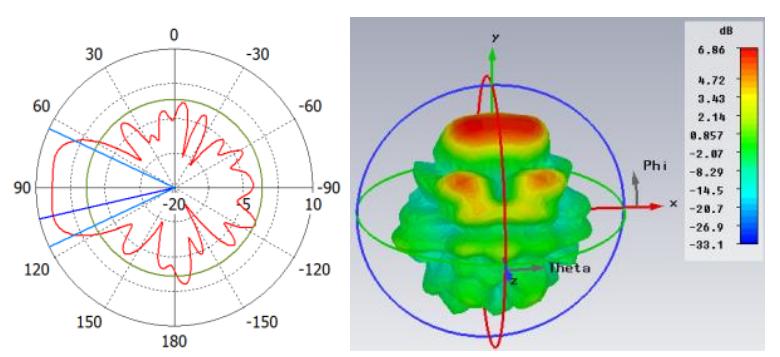

(d)

Fig 14: The gain in polar and 3D at different frequencies for $2 \times 2$ DASMA array with RT 5880 layer. (a) Frequency at $6 \mathrm{GHz}$-gain $4 \mathrm{~dB}$. (b) Frequency at 8.2GHz-gain 3.4dB.

(c) Frequency at 13GHz-gain 4dB. d) Frequency at 17GHz-gain $7 \mathrm{~dB}$

This new design has narrower band than the previous design; without stacked substrate; because of the mutual induction phenomena. For satellite bands operation this antenna can operate in C-band, X-band, Ku-band and K-band. To utilize most of these antenna bands, additional microwave devices should be used e.g. circulators, isolators, couplers and filters. Radiation patterns at multiple frequencies are presented in the polar and 3D forms shown in figure (14), giving an isoflux radiation shapes. In addition the antennas gain is increased by $+12 \mathrm{~dB}$ compared to DASMA where no stacked RT 5880 layer is employed as shown in Fig. (12).

\section{SERIAL ARRAY DIPOLE ARCHIMEDEAN SPIRAL MICROSTRIP ANTENNA 3x2 DASMA DESIGN AND GAIN ENHANCEMENT}

The modified design on the $2 \times 2$ dipole Archimedean spiral microstrip serial array antenna $2 \times 2$ DASMA is by adding another dipole in front of the original array in serial form forming $3 \times 2$ serial array, this design saves the same geometry of the patches. The first design prints the six patches on FR-4 stacked directly to the ground plane as shown in Fig. (15).
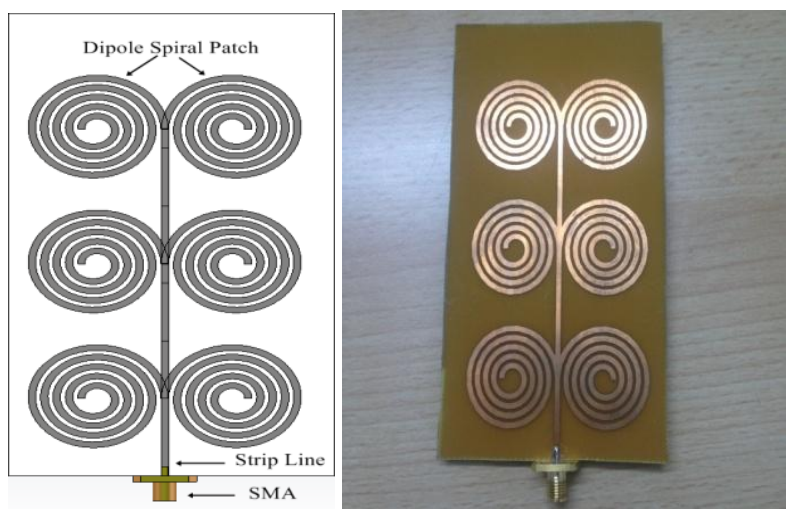

Fig 15: The cross section for $3 \times 2$ DASMA and its fabricated photo

After doing optimization and parameterization on the geometrical dimensions and taking into account the available material and fabrication techniques, the antenna with this design operate in the band between $7.5 \mathrm{GHz}$ to more than 40 $\mathrm{GHz}$ as shown in Fig. (16) [15]. Circular polarization achieved with this antenna as shown in figure (17) having an axial ratio below $3 \mathrm{~dB}$. The gain tread over the $0 \mathrm{~dB}$, as shown in figure (18) which need to increase to be suitable for onboard communications so stacked substrates gain enhancement technique can be used to raise the gain and radiation direction [16]-[20].

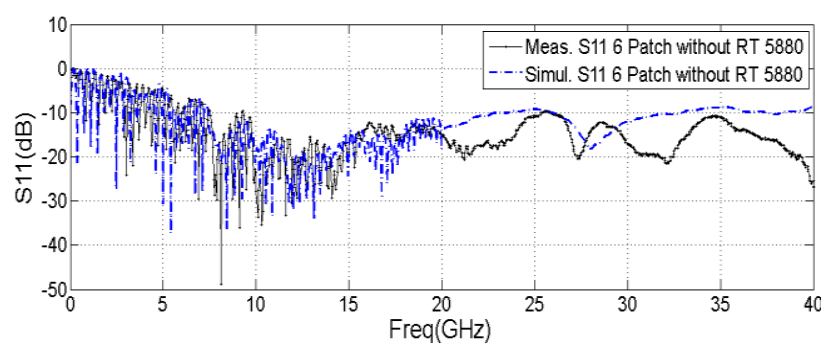

Fig 16: The return loss for 3x2 DASMA without RT 5880 layer

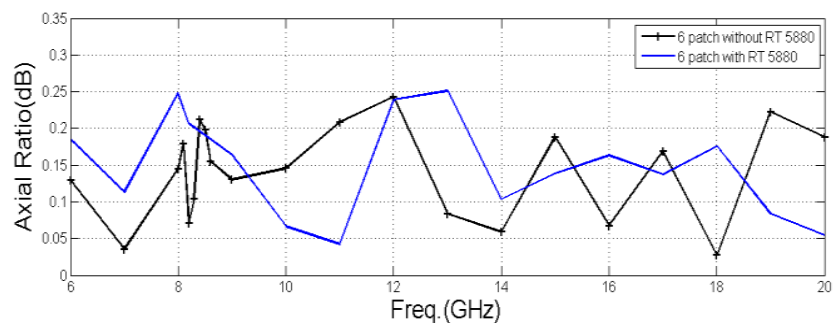

Fig 17: The axial ratio in broadband form for $3 \times 2$ DASMA with VS without RT 5880 layer

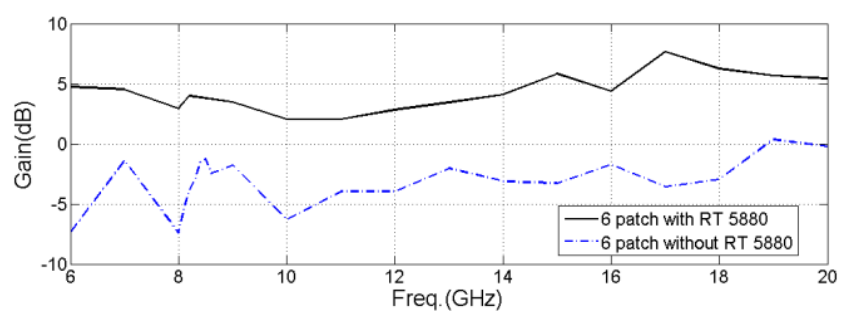

Fig 18: The gain in broadband form for $3 \times 2$ DASMA with VS without RT 5880 layer 
The modified design on $3 \times 2$ dipole Archimedean spiral microstrip serial array antenna DASMA saves the same geometry of the patches, stacked substrates technique employed in Fig. (5), which increases the thickness of FR-4 to $1.5 \mathrm{~mm}$ and adding RT $5880\left(\varepsilon_{\mathrm{r}}=2.2\right)$ with height $(0.508 \mathrm{~mm})$ as a new stacked material, thence the total substrate height between patches and ground plane reaches $2.008 \mathrm{~mm}$. This new modifications added to the previous antenna which can be operated in the band between 6 -to- $22 \mathrm{GHz}$ forming $16 \mathrm{GHz}$ of working band with circular polarization as shown in Fig. (19) and Fig. (17) respectively.

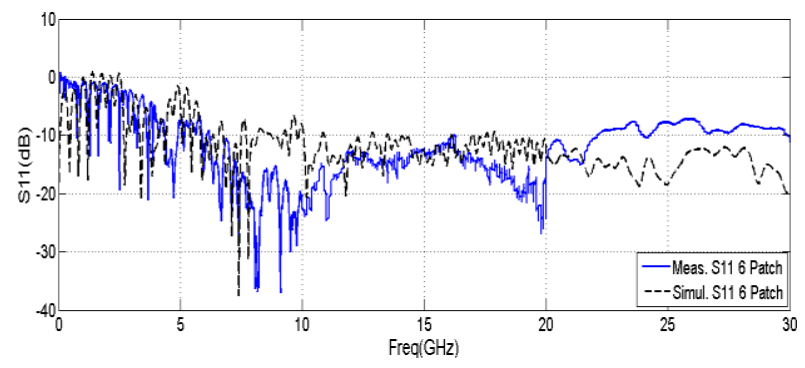

Fig 19: The RL for 3x2 DASMA with RT 5880 layer

This design had become narrower band than the previous design of $3 \times 2$ DASMA [15], because mutual induction phenomena takes place. For satellite bands operation this antenna can operate in C-band, X-band, Ku-band and K-band. To utilize the most utilization of these antenna bands, some of microwave devices can be used as circulators, isolators, couplers and filters. Circular polarization achieved with this antenna as shown in figure (17) having an axial ratio below $3 \mathrm{~dB}$. Radiation patterns in multiple frequencies presented in the polar and 3D forms as shown in figure (20), which is forming isoflux radiation shapes in some frequencies. In addition the operation of the antenna gain increases to be $7.8 \mathrm{dBi}$ as shown in Fig. (18).

\section{RESULTS DISCUSSION}

Starting with the normal Dipole Archimedean spiral microstrip antenna DASMA and it's modified by gain enhancement technique, the normal DASMA bandwidth extended from 7.5 -to- $24 \mathrm{GHz}$ and the achieved gain below OdB which considered as low gain as shown in Fig. (21) and Fig. (22) respectively.
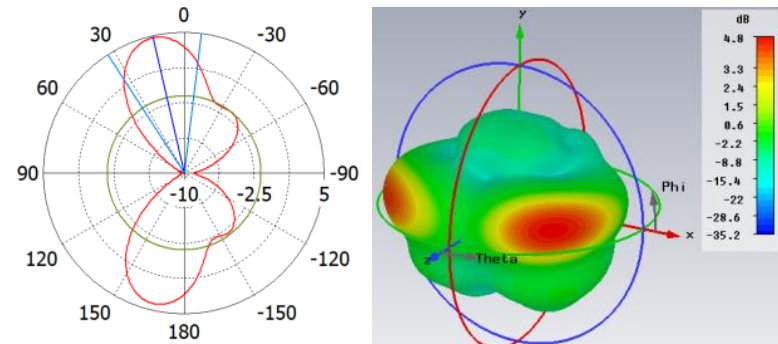

(a)

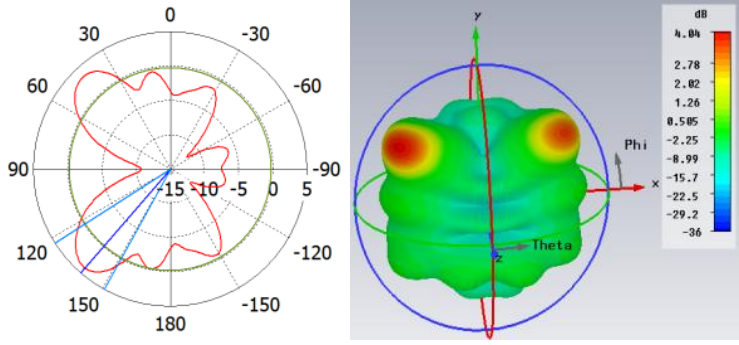

(b)
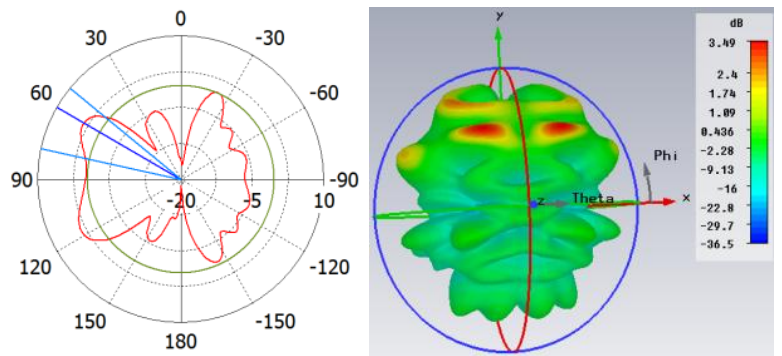

(c)

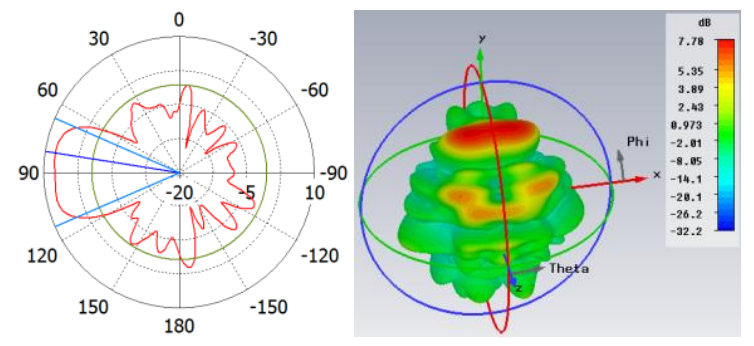

(d)

Fig 20: The gain in polar and 3D form in multiple frequencies as an examples for 3x2 DASMA serial array with RT 5880 layer, (a) at 6GHz-gain $4.8 \mathrm{~dB}$, (b) at 8.2GHz-gain $4 \mathrm{~dB}$, (c) at 13GHz-gain $3.5 \mathrm{~dB}$, (d) at $17 \mathrm{GHz}$ gain $7.8 \mathrm{~dB}$

When gain enhancement technique is used by adding stacked RT 5880 layer the gain increase but the bandwidth decreased that extended from $7.5-$ to- $15 \mathrm{GHz}$ as a continues bandwidth with gain enhancement by $+8 \mathrm{~dB}$ more than the normal DASMA. For further improvement in antenna bandwidth and for further enhancement in the antenna gain, another two patch added to the normal DASMA forming $2 \times 2$ Archimedean spiral serial array antenna, bandwidth extended from 7.5-to- $25 \mathrm{GHz}$ and the achieved gain increased by $+5 \mathrm{~dB}$ which achieved gain above $0 \mathrm{~dB}$ to be $1 \mathrm{dBi}$ as shown in Fig. (21) and Fig. (22) respectively which is good gain but onboard satellite communications need more gain. The key solution, stacked RT 5880 layer added to the normal array which increased the gain by $+10 \mathrm{~dB}$ more than the normal DASMA to be $7 \mathrm{dBi}$, but the bandwidth decreased that extended from 5.8-to-16 GHz, This bandwidth shrinkage also is caused by the mutual induction. Finally, $3 \times 2$ Archimedean spiral serial array antenna bandwidth extended from $7.5 \mathrm{GHz}$ to more than $40 \mathrm{GHz}$ and the achieved gain increased by $+6 \mathrm{~dB}$ relative to the normal DASMA which achieved gain above $0 \mathrm{~dB}$ to be $1 \mathrm{dBi}$ as shown in Fig. (21) and Fig. (22) respectively. Stacked RT 5880 layer added to the normal array which increased the gain by $+12 \mathrm{~dB}$ more than the normal 
DASMA to be $7.8 \mathrm{dBi}$, but the bandwidth decreased that extended from 6 -to- $22 \mathrm{GHz}$, which still more than the $2 \times 2$ DASMA, This bandwidth shrinkage also is caused by the mutual induction. For all the previous antennas are radiating in circular polarization as shown in Fig. (23), which is the advantage of spiral shapes.

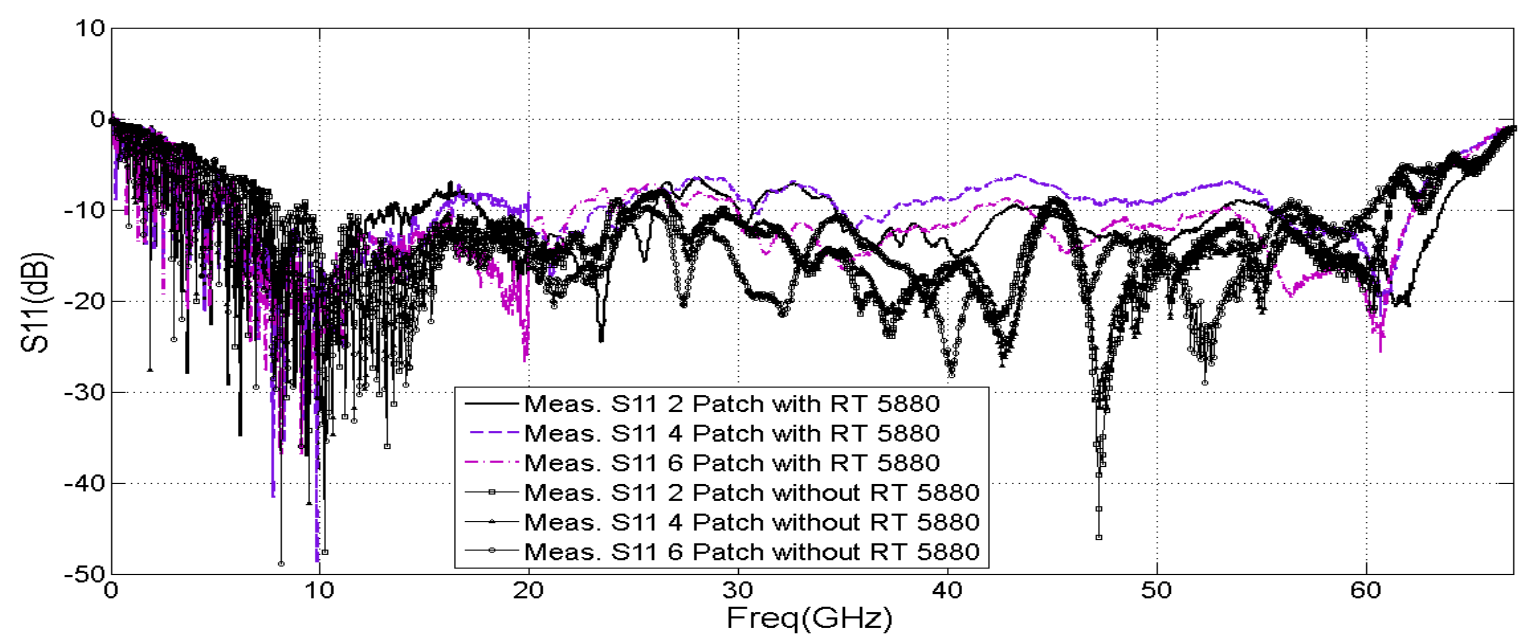

Fig 21: The return loss for all measured antennas with and without RT 5880

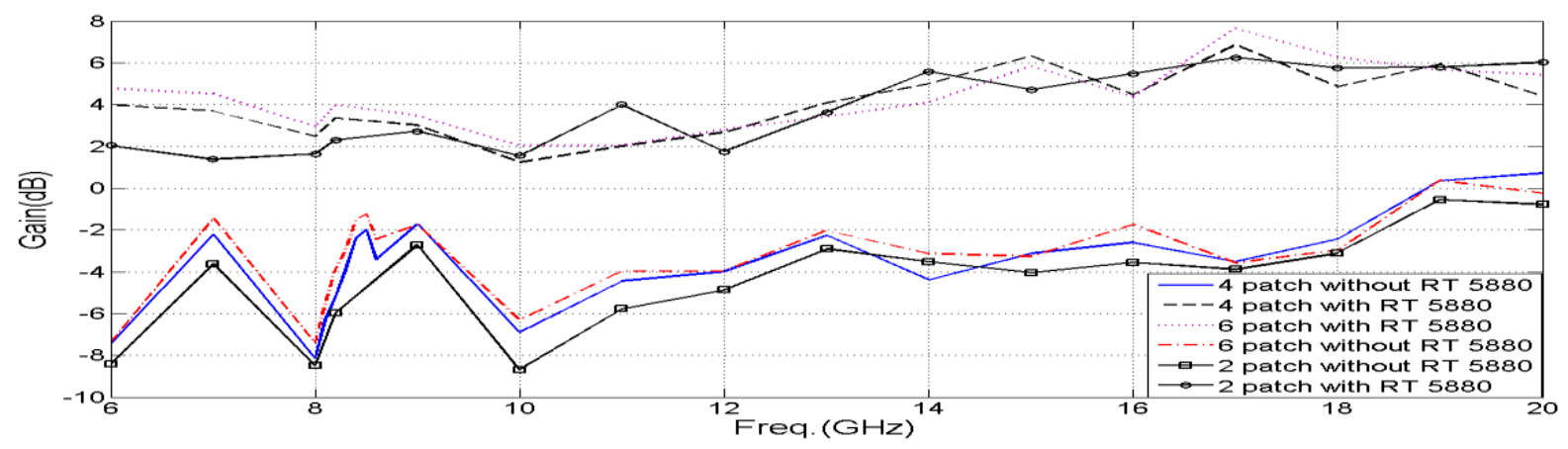

Fig 22: The gain in broadband form for all antennas with and without RT 5880 layer

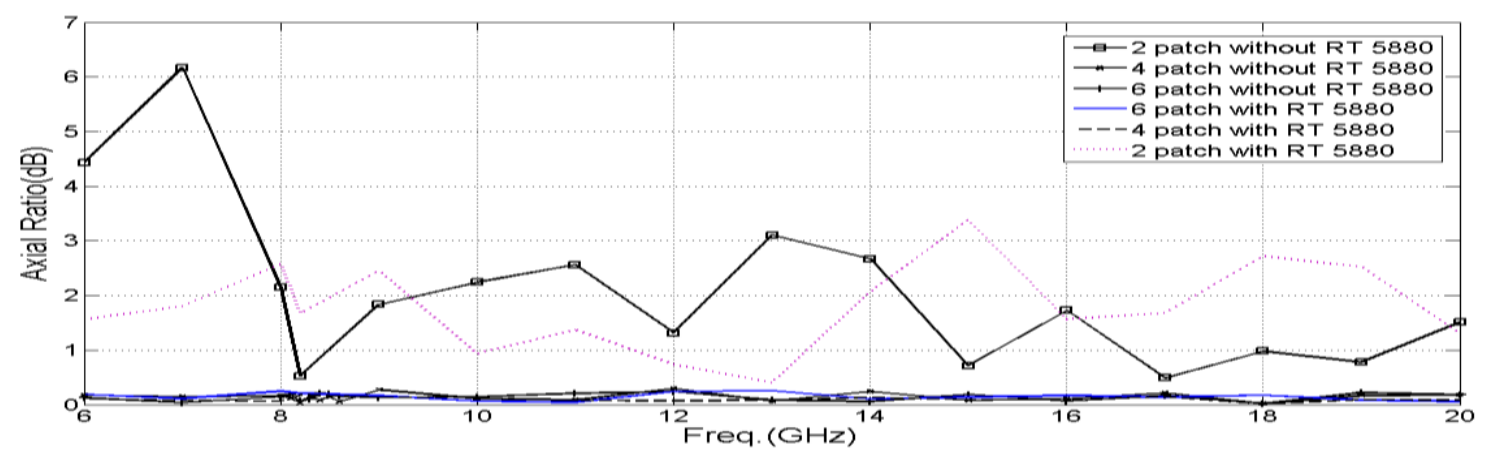

Fig 23: The axial Ratio in broadband form for all antennas with and without RT 5880 layer

\section{CONCLUSION AND FUTURE WORK}

An ultra-wide band UWB antenna design is presented using spiral shapes on FR-4 substrate without using cavities or absorbing materials. $3 \times 2$ Dipole Archimedean spiral microstrip serial array antenna DASMA achieve 6-to- $22 \mathrm{GHz}$ of working band and gain increases to $7.8 \mathrm{dBi}$. This antenna can be considered as UWB antenna in addition the use of RT 5880 increases gain, bandwidth and radiate in circular polarization. The gain enhancement technique by using stacked substrates as RT 5880 layer is outperforming the array technique or multiple patch when using FR-4 as a basic substrate. But when we added the two techniques with each other the result be in the best case. All of these features increase the visibility duration of the satellite and the ground station in multiple satellite bands. The future works will be focused on using other techniques to enhance the gain and overcome the mutual induction.

\section{REFERENCES}

[1] Rumsey, V.H., "Frequency independent antennas," IRE National Convention Record, vol. 5, pt.1, pp. 114-118, 1957.

[2] Dyson, J.D., "The equiangular spiral antenna," IRE Transactions on Antennas and Propagation, vol. AP-7, 
pp. 181-187, April 1959.

[3] Dyson, J.D., "The unidirectional equiangular spiral antenna," IRE Transactions on Antennas and Propagation, vol. AP-7, pp. 329-334, October 1959.

[4] Bawer, R. and Wolfe, J.J., "The spiral antenna," IRE National Convention Record, pp. 84-89, New York, March 1960.

[5] Curtis W.L., "Spiral antennas," IRE Transactions on Antennas and Propagation, Vol. AP-8, pp. 298-306, May 1960.

[6] I J.Bahl, P.Bhartia, "Microstrip Antennas", Artech House, INC., 1980.

[7] C.A. Balanis, "Antenna Theory Analysis and Design ", John Wiley \& Sons, INC., NY, 1997.

[8] R. Garg, P. Bhartia, I. Bahl, A. Ittipiboon, "Microstrip Antenna Design Handbook", Artech House, INC., 2001.

[9] GirishKumar, K. P. Ray, "Broadband Microstrip Antennas", Artech House, INC., 2003.

[10] D.M. Pozar, "Microwave Engineering", 3rd edition, John Wiely \& sons, INC., 2003.

[11] H. Nakano and K. Hirose, "Experimental investigation of slot type dual spiral antenna fed by a microstrip line," AP-S. Digest IEEE 1989.

[12] Nakano, H., Nogami, K, Arai, S., Mimaki, H, and Yamauchi, J., "A spiral antenna backed by a conducting plane reflector," IEEE Transactions on Antennas and Propagation, pp. 791-796, June 1986.

[13] Wu, S-C., "Analysis and design of conductor backed square Archimedean spiral antennas," Electromagnetics, vol. 14, pp. 305-318, 1994.

[14] Numberger, M.W. and Volakis, J.L., "Extremely broadband slot spiral antenna with shallow reflecting cavities," Electromagnetics, vol 20, pp. 357- 376, 2000.

[15] F. Bilotti, A. Alu, L. Vegni, P. Baldonero, "Single Arm Hexagonal Spiral Element for Ultra Wide Band Phased Antenna Arrays ", 18th International Conference on Applied Electromagnetics and Communications, ICECom 2005.

[16] F. Klefenz and A. Dreher, "Aperture-Coupled Stacked Microstrip Antenna with Dual Polarization and Low Back-Radiation for X-Band SAR Applications" IEEE 2000.

[17] Wei Wang, Shun-Shi Zhong and Xian-Ling Liang, "A dual-polarized stacked microstrip antenna subarray for X-band SAR application," IEEE 2004.

[18] James A. Nessel, Afroz Zaman, Richard Q. Lee, and Kevin Lambert "Demonstration of an X-Band Multilayer Yagi-Like Microstrip Patch Antenna with High Directivity and Large Bandwidth,” IEEE 2005.

[19] Abbas Ali Heidari, Mojtaba Simrooni and Mansor Nakhkash, "Analysis and Design of an X-Band Microstrip Patch Array Antenna for ESM Application", IEEE 2007.

[20] Mohamed A. Elmansouri, James B. Bargeron, and Dejan S. Filipovic, "Simply-Fed Four-Arm Spiral-Helix Antenna," IEEE Transactions on Antennas and Propagation, Vol. 62, No. 9, September 2014. 